\title{
Von Neumann-Morgenstern solutions in the assignment market
}

\author{
Marina Núñez ${ }^{\mathrm{a}, 1}$, Carles Rafels ${ }^{\mathrm{a}, 1}$ \\ ${ }^{a}$ Departament de Matemàtica Econòmica, Financera i Actuarial, Universitat de \\ Barcelona, Av. Diagonal, 690; 08034 Barcelona, Spain
}

\begin{abstract}
The existence of von Neumann-Morgenstern solutions (stable sets) for assignment games has been an unsolved question since Shapley and Shubik [11]. For each optimal matching between buyers and sellers, Shubik [12] proposed considering the union of the core of the game and the core of the subgames that are compatible with this matching. We prove in the present paper that this set is the unique stable set for the assignment game that excludes thirdparty payments with respect to a fixed optimal matching. Moreover, the stable sets that we characterize, as well as any other stable set of the assignment game, have a lattice structure with respect to the same partial order usually defined on the core.
\end{abstract}

Keywords: assignment game, core, von Neumann-Morgenstern stable set $J E L: \mathrm{C} 71$

\footnotetext{
Email addresses: mnunez@ub.edu (Marina Núñez), crafels@ub.edu (Carles Rafels)

${ }^{1}$ The authors acknowledge the support from research grant ECO2011-22765 (Ministerio de Ciencia e Innovación and FEDER).
} 


\section{Introduction}

The assignment game was introduced by Shapley and Shubik [11] as a cooperative model for a market with buyers and sellers. Each seller has one indivisible good or "object" to sell, and each buyer wants to buy at most one object. Objects are distinct and buyers may value them differently. The valuation matrix summarizes the profit that each mixed-pair can attain by the trade of the object between them. The worth of the grand coalition is the total profit that can be obtained by optimally matching buyers to sellers, and the worth of any other coalition is obtained in a similar way, just restricting attention to the corresponding submatrix.

Von Neumann and Morgenstern [14] introduced the first solution concept for general cooperative games, based on a dominance relation between imputations, i.e. individually rational allocations of the worth of the grand coalition. One imputation dominates another if each member of a given coalition gets strictly more in the first imputation than in the second one, and these payoffs are feasible for this coalition.

A von Neumann-Morgenstern solution (a stable set) $V$ is a set of imputations satisfying (i) internal stability: no coalition objects to an imputation in $V$ by proposing a dominating imputation in $V$, and (ii) external stability: each imputation outside $V$ is objected to by some coalition that proposes a dominating imputation in $V$. According to von Neumann and Morgenstern, each stable set represents a standard of behavior. Trivially, each stable set of a game contains the core, that is, the set of undominated imputations (Gillies, [3]). Stable sets are difficult to characterize (Aumann, [1]) and they may not exist. Lucas ([5]) provides examples of games with no stable set. 
Although the core, in preference to the stable sets, has become the most common solution applied to cooperative games, we argue in the present paper that the core is not sufficient to analyze all the bargaining possibilities of the agents in an assignment market.

Assume that, after an optimal matching has been agreed on, one buyer decides to pay nothing for his assigned good. His assigned seller turns to the remaining agents but, if he finds himself unmatched in the resulting subgame, we may assume the buyer can obtain the object at a null price. Once this buyer has left the market, the remaining agents can share the profit of their partnership according to a core element of the subgame. This imputation, which may be outside the core of the initial game, is undominated by any core element. We show that imputations of this $\mathrm{kind}^{2}$ form a stable set of the assignment game.

Consequently, our main result is the proof of the existence of stable sets for the assignment game. The proof is constructive and the stable set is formed by particular imputations of the sort described in the paragraph above. We derive one stable set associated with each optimal matching of the market, and this stable set is the only one that excludes third-party payments with respect to this optimal matching.

The stable sets we characterize are very closely connected to the notion of the core, since they enlarge the core by taking into consideration the core elements of some submarkets. By doing so, these stable sets overcome some drawbacks the core alone may present. When we choose the core as a solu-

\footnotetext{
${ }^{2}$ See Definition 3.1 and the concept of extended core in (4).
} 
tion we exclude an imputation if it is dominated by some other imputation, although this other imputation may also be outside the core. An example of this situation is provided by the "glove market", a symmetric assignment market with constant valuation matrix. In a glove market with fewer sellers than buyers, the unique core allocation gives all the profit to the sellers, while buyers get zero, not taking into account the fact that sellers need the cooperation of at least a buyer to make any profit. Moreover, this unique core allocation does not dominate any other imputation. Similar situations appear in general assignment markets.

In [10], Shapley describes most of the von Neumann-Morgenstern stable sets of a glove market. In Shubik [12], and also in some personal notes of Shapley, ${ }^{3}$ a set of imputations is claimed as a stable set for the assignment game. However, this claim is not accompanied by a complete proof. The present paper closes this gap and establishes the existence of von NeumannMorgenstern stable sets for assignment games.

The set defined by Shubik consists of the union of the core of the assignment market and the cores of some selected submarkets that are compatible with an optimal matching which has been fixed beforehand. The stability of this set is proved in Section 3 (part of the proof is consigned to the supplemental material). In Section 2 the basic definitions regarding assignment games are given. Section 4 concludes with some remarks.

\footnotetext{
${ }^{3}$ We thank professor T. Solymosi for providing us with these notes.
} 


\section{Preliminaries}

In an assignment market, a product that comes in indivisible units is on sale, and each agent either supplies or demands exactly one unit. Thus, the set of agents is partitioned into a finite set of buyers $M$ and a finite set of sellers $M^{\prime}$. The profit each mixed-pair $(i, j) \in M \times M^{\prime}$ can attain is given by the valuation matrix $A=\left(a_{i j}\right)_{(i, j) \in M \times M^{\prime}}$.

A matching for the market $\left(M, M^{\prime}, A\right)$ is a bijection $\mu$ between a subset of $M$ and a subset of $M^{\prime}$. We denote by $\mathcal{M}\left(M, M^{\prime}\right)$ this set of matchings. An optimal matching is a matching $\mu \in \mathcal{M}\left(M, M^{\prime}\right)$ such that $\sum_{(i, j) \in \mu} a_{i j} \geq$ $\sum_{(i, j) \in \mu^{\prime}} a_{i j}$ for all $\mu^{\prime} \in \mathcal{M}\left(M, M^{\prime}\right)$. We denote by $\mathcal{M}_{A}^{*}\left(M, M^{\prime}\right)$ the set of optimal matchings for $\left(M, M^{\prime}, A\right)$. If $(i, j) \in \mu$ we say that $i$ and $j$ are matched (or assigned) by $\mu$ and we also write $j=\mu(i)$ and $i=\mu^{-1}(j)$. If for some buyer $i \in M$ there is no $j \in M^{\prime}$ such that $(i, j) \in \mu$ we say that $i$ is unmatched (or unassigned) by $\mu$ (and similarly for sellers).

Given $S \subseteq M$ and $T \subseteq M^{\prime}$, we denote by $\mu_{\mid S \times T}$ the restriction of $\mu \in$ $\mathcal{M}\left(M, M^{\prime}\right)$ to the pairs in $S \times T$. Moreover, we denote by $\mathcal{M}(S, T)$ and $\mathcal{M}_{A}^{*}(S, T)$ the set of matchings and optimal matchings of the submarket $\left(S, T, A_{\mid S \times T}\right)$ defined by the subset $S$ of buyers, the subset $T$ of sellers and the restriction of $A$ to $S \times T$. If $S=\emptyset$ or $T=\emptyset$, then the only possible matching in $\mathcal{M}(S, T)$ is $\mu=\emptyset$ and by convention $\sum_{(i, j) \in \emptyset} a_{i j}=0$.

The cooperative game $\left(M \cup M^{\prime}, w_{A}\right)$ for the assignment market is defined by the set of players $M \cup M^{\prime}$ and the characteristic function $w_{A}(S \cup T)=$ $\max \left\{\sum_{(i, j) \in \mu} a_{i j} \mid \mu \in \mathcal{M}(S, T)\right\}$, for all $S \subseteq M$ and $T \subseteq M^{\prime}$. The assignment game is square if and only if its valuation matrix is square, that is, if there are as many buyers as sellers. 
Given an assignment game $\left(M \cup M^{\prime}, w_{A}\right)$, a payoff vector is $(u, v) \in$ $\mathbb{R}^{M} \times \mathbb{R}^{M^{\prime}}$, where $u_{i}$ stands for the payoff to buyer $i \in M$ and $v_{j}$ stands for the payoff to seller $j \in M^{\prime}$. An imputation is a non-negative payoff vector that is efficient, $\sum_{i \in M} u_{i}+\sum_{j \in M^{\prime}} v_{j}=w_{A}\left(M \cup M^{\prime}\right)$. We denote by $I\left(w_{A}\right)$ the set of imputations of the assignment game $\left(M \cup M^{\prime}, w_{A}\right)$.

A binary relation is defined on the set of imputations. If $(u, v),\left(u^{\prime}, v^{\prime}\right) \in$ $I\left(w_{A}\right)$, we say $\left(u^{\prime}, v^{\prime}\right)$ dominates $(u, v)$, and write $\left(u^{\prime}, v^{\prime}\right) d o m^{w_{A}}(u, v)$, if and only if there exists $(i, j) \in M \times M^{\prime}$ such that $\left(u^{\prime}, v^{\prime}\right) \operatorname{dom}_{\{i, j\}}^{w_{A}}(u, v)$, that is to say, $u_{i}^{\prime}>u_{i}, v_{j}^{\prime}>v_{j}$ and $u_{i}^{\prime}+v_{j}^{\prime} \leq a_{i j} .{ }^{4}$ When no confusion regarding the game arises, we simply write $\left(u^{\prime}, v^{\prime}\right) \operatorname{dom}(u, v)$.

A subset $V$ of imputations is a stable set (von Neumann and Morgenstern, [14]) if it is internally stable (for all $(u, v),\left(u^{\prime}, v^{\prime}\right) \in V,\left(u^{\prime}, v^{\prime}\right)$ does not dominate $(u, v)$ ) and externally stable (for all $(u, v) \in I\left(w_{A}\right) \backslash V$, there exists $\left(u^{\prime}, v^{\prime}\right) \in V$ such that $\left.\left(u^{\prime}, v^{\prime}\right) \operatorname{dom}(u, v)\right)$.

The core is also defined by means of the above dominance relation as the set of undominated imputations. Shapley and Shubik ( [11]) prove that an assignment game $\left(M \cup M^{\prime}, w_{A}\right)$ always has a non-empty core. Moreover, with any optimal matching $\mu \in \mathcal{M}_{A}^{*}\left(M, M^{\prime}\right)$ fixed, a non-negative payoff vector $(u, v) \in \mathbb{R}_{+}^{M} \times \mathbb{R}_{+}^{M^{\prime}}$ is in the core of $\left(M \cup M^{\prime}, w_{A}\right)$ if and only if $u_{i}+v_{j} \geq a_{i j}$ for all $(i, j) \in M \times M^{\prime}, u_{i}+v_{j}=a_{i j}$ for all $(i, j) \in \mu$, and all agents unmatched by $\mu$ get a null payoff.

Two particular core allocations are the buyers-optimal core allocation $\left(\bar{u}^{A}, \underline{v}^{A}\right)$ where each buyer obtains her maximum core payoff, and each seller

\footnotetext{
${ }^{4}$ For assignment games, this dominance relation via mixed-pair coalitions is equivalent to von Neumann and Morgenstern's classical dominance relation.
} 
his minimum one, and the sellers-optimal core allocation $\left(\underline{u}^{A}, \bar{v}^{A}\right)$ where each seller obtains his maximum core payoff and each buyer her minimum one.

Solymosi and Raghavan ([13]) prove that the core of an assignment game $\left(M \cup M^{\prime}, w_{A}\right)$ is a von Neumann-Morgenstern stable set if and only if $\underline{u}_{i}^{A}=0$ for all $i \in M$ and $\underline{v}_{j}^{A}=0$ for all $j \in M^{\prime}$. When the assignment matrix is square, this is equivalent to saying that each matrix entry corresponding to an optimal pair is a row and column maximum.

In Núñez and Rafels [6], it is shown that given an assignment game $(M \cup$ $\left.M^{\prime}, w_{A}\right)$, there exists another (and unique) assignment game $\left(M \cup M^{\prime}, w_{A^{r}}\right)$ with the same core, $C\left(w_{A}\right)=C\left(w_{A^{r}}\right)$, and the property that for all $(i, j) \in$ $M \times M^{\prime}$ there exists $(x, y) \in C\left(w_{A^{r}}\right)=C\left(w_{A}\right)$ such that $x_{i}+y_{j}=a_{i j}^{r}$. This game is defined by $a_{i j}^{r}=\min _{(u, v) \in C\left(w_{A}\right)} u_{i}+v_{j}$ for all $(i, j) \in M \times M^{\prime}$, and matrix $A^{r}$ is the buyer-seller exact representative of matrix $A$. When there are as many buyers as sellers and $\mu \in \mathcal{M}_{A}^{*}\left(M, M^{\prime}\right)$ is an optimal matching that does not leave agents unassigned, $A^{r}$ can also be obtained only in terms of the entries of the original valuation matrix:

$a_{i j}^{r}=\max _{\left\{k_{1}, \ldots, k_{r}\right\} \subseteq M \backslash\left\{i, \mu^{-1}(j)\right\}}\left\{a_{i \mu\left(k_{1}\right)}+a_{k_{1} \mu\left(k_{2}\right)}+\cdots+a_{k_{r} j}-a_{k_{1} \mu\left(k_{1}\right)}-\cdots-a_{k_{r} \mu\left(k_{r}\right)}\right\}$.

Expression (1) will be repeatedly used in the proof of the main theorem.

Finally, the matrix $A^{e}$ is defined, for all $(i, j) \in M \times M^{\prime}$, by

$$
a_{i j}^{e}=a_{i j}^{r}-\underline{u}_{i}^{A}-\underline{v}_{j}^{A} .
$$

This matrix $A^{e}$ is introduced in Núñez and Rafels [7] and its associated assignment game $\left(M \cup M^{\prime}, w_{A^{e}}\right)$ has two properties that are relevant for our purposes: $C\left(w_{A}\right)=\left\{\left(\underline{u}^{A}, \underline{v}^{A}\right)\right\}+C\left(w_{A^{e}}\right)$ and $C\left(w_{A^{e}}\right)$ is the unique stable set 
of the game $\left(M \cup M^{\prime}, w_{A^{e}}\right)$.

In the paper, we will mainly focus on those imputations where, as happens to the core, transfers of money are only made between matched agents. Given an assignment game $\left(M \cup M^{\prime}, w_{A}\right)$ and an optimal matching $\mu \in \mathcal{M}_{A}^{*}\left(M, M^{\prime}\right)$, $(u, v) \in \mathbb{R}_{+}^{M} \times \mathbb{R}_{+}^{M^{\prime}}$ belongs to the $\mu$-principal section $B^{\mu}\left(w_{A}\right)$ if and only if $u_{i}+$ $v_{j}=a_{i j}$ for all $(i, j) \in \mu$, while unmatched agents get zero. The domination relation between imputations in the $\mu$-principal section is preserved if we make the market square by adding dummy agents on the short side (that is, zero row/columns to the matrix).

\section{The main result}

In this section we define the notion of a compatible subgame, introduced in Shubik [12], and prove how the cores of these subgames determine a stable set for the assignment game.

Definition 3.1. Let $\left(M \cup M^{\prime}, w_{A}\right)$ be an assignment game, $\mu \in \mathcal{M}_{A}^{*}\left(M, M^{\prime}\right)$ and let $I \subseteq M$ and $J \subseteq M^{\prime}$. The subgame $\left.\left((M \backslash I) \cup\left(M^{\prime} \backslash J\right)\right), w_{A_{\mid(M \backslash I) \times\left(M^{\prime} \backslash J\right)}}\right)$ is a $\mu$-compatible subgame of $\left(M \cup M^{\prime}, w_{A}\right)$ if and only if

$$
w_{A}\left((M \backslash I) \cup\left(M^{\prime} \backslash J\right)\right)+\sum_{\substack{i \in I \\ i \text { assigned by } \mu}} a_{i \mu(i)}+\sum_{\substack{j \in J \\ j \text { assigned by } \mu}} a_{\mu^{-1}(j) j}=w_{A}\left(M \cup M^{\prime}\right) .
$$

Notice that, for any assignment game and any optimal matching $\mu$, there always exist $\mu$-compatible subgames ${ }^{5}$ (just take $I=J=\emptyset$ ).

We write $w_{A_{-I \cup J}}$ for the characteristic function of the subgame with player set $(M \backslash I) \cup\left(M^{\prime} \backslash J\right)$. To say that $\left((M \backslash I) \cup\left(M^{\prime} \backslash J\right), w_{A_{-I \cup J}}\right)$ is a $\mu$-compatible

\footnotetext{
${ }^{5}$ Properties of the $\mu$-compatible subgames can be found in the supplemental material.
} 
subgame is equivalent to saying that the restriction of $\mu$ to $(M \backslash I) \times\left(M^{\prime} \backslash J\right)$ is still optimal for the resulting submarket and whenever an agent has been excluded (let us say $i \in I$ ), his partner remains in the submarket $(\mu(i) \notin J)$, unless $a_{i \mu(i)}=0$.

Given an assignment game $\left(M \cup M^{\prime}, w_{A}\right)$ and $\mu \in \mathcal{M}_{A}^{*}\left(M, M^{\prime}\right)$, if for some $I \subseteq M$ and $J \subseteq M^{\prime}$ the subgame $\left((M \backslash I) \cup\left(M^{\prime} \backslash J\right), w_{A_{-I \cup J}}\right)$ is $\mu$-compatible, then $\hat{C}\left(w_{A_{-I \cup J}}\right)$ is its extended core,

$\hat{C}\left(w_{A_{-I \cup J}}\right)=\left\{\begin{array}{l|l}(u, v) \in B^{\mu}\left(w_{A}\right) & \begin{array}{l}\left(u_{-I}, v_{-J}\right) \in C\left(w_{A_{-I \cup J}}\right), \\ u_{i}=a_{i \mu(i)} \text { for all } i \in I \text { assigned by } \mu, \\ v_{j}=a_{\mu^{-1}(j) j} \text { for all } j \in J \text { assigned by } \mu\end{array}\end{array}\right\}$

The claim in Shubik [12] is that, for any optimal matching $\mu$, we should add to the core of the game $\left(M \cup M^{\prime}, w_{A}\right)$ the extended cores of all its $\mu$ compatible subgames to obtain a stable set. Let us denote by $\mathcal{C}_{A}^{\mu}$ the set of pairs $(I, J), I \subseteq M$ and $J \subseteq M^{\prime}$, such that $\left((M \backslash I) \cup\left(M^{\prime} \backslash J\right), w_{A_{-I \cup J}}\right)$ is a $\mu$-compatible subgame of $\left(M \cup M^{\prime}, w_{A}\right)$.

Theorem 3.1. Let $\left(M \cup M^{\prime}, w_{A}\right)$ be an assignment game and $\mu \in \mathcal{M}_{A}^{*}\left(M, M^{\prime}\right)$ an optimal matching. The set

$$
V^{\mu}\left(w_{A}\right)=\bigcup_{(I, J) \in \mathcal{C}_{A}^{\mu}} \hat{C}\left(w_{A_{-I \cup J}}\right)
$$

is a von Neumann-Morgenstern stable set of $\left(M \cup M^{\prime}, w_{A}\right)$.

Proof. On page 207 of Shubik [12] it is proved that $V^{\mu}\left(w_{A}\right)$ is undominated by any imputation in the $\mu$-principal section, which implies the internal stability of $V^{\mu}\left(w_{A}\right)$, and that any imputation outside the $\mu$-principal section is dominated by an element in $V^{\mu}\left(w_{A}\right)$. 
We will complete the proof of the external stability of $V^{\mu}\left(w_{A}\right)$ by stating and proving three claims. Before that, and since $C\left(w_{A}\right) \subseteq V^{\mu}\left(w_{A}\right)$, we define the subset $R^{\mu}\left(w_{A}\right)$ of the $\mu$-principal section formed by allocations in which each agent is paid an amount in between his/her minimum and maximum core payoff: given an assignment game $\left(M \cup M^{\prime}, w_{A}\right)$ and $\mu \in \mathcal{M}_{A}^{*}\left(M, M^{\prime}\right)$,

$$
R^{\mu}\left(w_{A}\right)=\left\{(u, v) \in B^{\mu}\left(w_{A}\right) \mid \underline{u}_{i}^{A} \leq u_{i} \leq \bar{u}_{i}^{A} \text { for all } i \in M\right\} .
$$

Consequently, for all $(u, v) \in R^{\mu}\left(w_{A}\right)$ it also holds $\underline{v}_{j}^{A} \leq v_{j} \leq \bar{v}_{j}^{A}$ for all $j \in M^{\prime}$.

Notice that $C\left(w_{A}\right) \subseteq R^{\mu}\left(w_{A}\right) \subseteq B^{\mu}\left(w_{A}\right) \subseteq I\left(w_{A}\right)$. We first claim that any imputation in $R^{\mu}\left(w_{A}\right) \backslash V^{\mu}\left(w_{A}\right)$ is dominated by a core allocation of $\left(M \cup M^{\prime}, w_{A}\right)$.

Claim 3.1. Let $\left(M \cup M^{\prime}, w_{A}\right)$ be an assignment game and $\mu \in \mathcal{M}_{A}^{*}\left(M, M^{\prime}\right)$. For all $(u, v) \in R^{\mu}\left(w_{A}\right) \backslash V^{\mu}\left(w_{A}\right)$, there exists $\left(u^{\prime}, v^{\prime}\right) \in C\left(w_{A}\right)$ such that $\left(u^{\prime}, v^{\prime}\right) \operatorname{dom}^{w_{A}}(u, v)$.

Proof of Claim 3.1 We may assume without loss of generality that $A$ is a square matrix, and that $\mu$ does not leave agents unassigned, since both $C\left(w_{A}\right)$ and $R^{\mu}\left(w_{A}\right)$ allow for this simplification. Let $\left(M \cup M^{\prime}, w_{A^{e}}\right)$ be the related exact assignment game and remember from (2) that $a_{i j}^{e}=a_{i j}^{r}-\underline{u}_{i}^{A}-$ $\underline{v}_{j}^{A}$ for all $i \in M, j \in M^{\prime}$. Notice first that $(u, v) \in R^{\mu}\left(w_{A}\right) \backslash V^{\mu}\left(w_{A}\right)$ implies $(u, v) \in R^{\mu}\left(w_{A}\right) \backslash C\left(w_{A}\right)$. Since $\mu$ is also an optimal matching for $\left(M, M^{\prime}, A^{e}\right)$ and for $\left(M, M^{\prime}, A^{r}\right)$, and $C\left(w_{A}\right)=\left\{\left(\underline{u}^{A}, \underline{v}^{A}\right)\right\}+C\left(w_{A^{e}}\right)$, given $(u, v) \in R^{\mu}\left(w_{A}\right) \backslash C\left(w_{A}\right)$, we have $\left(u-\underline{u}^{A}, v-\underline{v}^{A}\right) \in B^{\mu}\left(w_{A^{e}}\right) \backslash C\left(w_{A^{e}}\right)$.

Since $\left(M \cup M^{\prime}, w_{A^{e}}\right)$ has a stable core, there exist $\left(u^{\prime \prime}, v^{\prime \prime}\right) \in C\left(w_{A^{e}}\right)$ and $\left(i^{*}, j^{*}\right) \in M \times M^{\prime}$ such that $\left(u^{\prime \prime}, v^{\prime \prime}\right) \operatorname{dom}_{\left\{i^{*}, j^{*}\right\}}^{w_{e}}\left(u-\underline{u}^{A}, v-\underline{v}^{A}\right)$. 
This means that

$$
u_{i^{*}}^{\prime \prime}>u_{i^{*}}-\underline{u}_{i^{*}}^{A}, \quad v_{j^{*}}^{\prime \prime}>v_{j^{*}}-\underline{v}_{j^{*}}^{A}, \quad \text { and } \quad u_{i^{*}}^{\prime \prime}+v_{j^{*}}^{\prime \prime} \leq a_{i^{*} j^{*}}^{e} .
$$

Let us now define

$$
(\tilde{u}, \tilde{v})=\left(u^{\prime \prime}, v^{\prime \prime}\right)+\left(\underline{u}^{A}, \underline{v}^{A}\right) .
$$

Notice that $(\tilde{u}, \tilde{v}) \in C\left(w_{A}\right)=C\left(w_{A^{r}}\right)$ and, by $(7)$,

$$
\tilde{u}_{i^{*}}=u_{i^{*}}^{\prime \prime}+\underline{u}_{i^{*}}^{A}>u_{i^{*}}, \quad \tilde{v}_{j^{*}}=v_{j^{*}}^{\prime \prime}+\underline{v}_{j^{*}}^{A}>v_{j^{*}} \text { and } \tilde{u}_{i^{*}}+\tilde{v}_{j^{*}} \leq a_{i^{*} j^{*}}^{r},
$$

which implies $(\tilde{u}, \tilde{v}) \operatorname{dom}_{\left\{i^{*}, j^{*}\right\}}^{w_{A^{r}}}(u, v)$. We must prove that $(u, v)$ is also dominated by a core allocation in terms of the game $w_{A}$ instead of $w_{A^{r}}$. From (1), either $a_{i^{*} j^{*}}^{r}=a_{i^{*} j^{*}}$ and we are done, or

$\tilde{u}_{i^{*}}+\tilde{v}_{j^{*}} \leq a_{i^{*} j^{*}}^{r}=a_{i^{*} \mu\left(i_{1}\right)}+a_{i_{1} \mu\left(i_{2}\right)}+\cdots+a_{i_{r} j^{*}}-a_{i_{1} \mu\left(i_{1}\right)}-a_{i_{2} \mu\left(i_{2}\right)}-\cdots-a_{i_{r} \mu\left(i_{r}\right)}$ for some distinct $i_{1}, i_{2}, \ldots, i_{r} \in M \backslash\left\{i^{*}, \mu^{-1}\left(j^{*}\right)\right\}$.

In this case, since $(\tilde{u}, \tilde{v}) \in B^{\mu}\left(w_{A}\right), \tilde{u}_{i_{l}}+\tilde{v}_{\mu\left(i_{l}\right)}=a_{i_{l} \mu\left(i_{l}\right)}$ for $l \in\{1,2, \ldots, r\}$ we obtain

$$
\tilde{u}_{i^{*}}+\tilde{v}_{j^{*}}+\tilde{u}_{i_{1}}+\tilde{v}_{\mu\left(i_{1}\right)}+\cdots+\tilde{u}_{i_{r}}+\tilde{v}_{\mu\left(i_{r}\right)} \leq a_{i^{*} \mu\left(i_{1}\right)}+a_{i_{1} \mu\left(i_{2}\right)}+\cdots+a_{i_{r} j^{*}} .
$$

Together with $(\tilde{u}, \tilde{v}) \in C\left(w_{A}\right)$, this implies $\tilde{u}_{i^{*}}+\tilde{v}_{\mu\left(i_{1}\right)}=a_{i^{*} \mu\left(i_{1}\right)}, \tilde{u}_{i_{l}}+\tilde{v}_{\mu\left(i_{l+1}\right)}=$ $a_{i_{l} \mu\left(i_{l+1}\right)}$ for all $l \in\{1,2, \ldots, r-1\}$ and $\tilde{u}_{i_{r}}+\tilde{v}_{j^{*}}=a_{i_{r} j^{*}}$.

- If $\tilde{v}_{\mu\left(i_{1}\right)}>v_{\mu\left(i_{1}\right)}$, and since, by (9), $\tilde{u}_{i^{*}}>u_{i^{*}}$, we are done because $(\tilde{u}, \tilde{v}) \operatorname{dom}_{\left\{i^{*}, \mu\left(i_{1}\right)\right\}}^{w_{A}}(u, v)$. Otherwise, that is $\tilde{v}_{\mu\left(i_{1}\right)} \leq v_{\mu\left(i_{1}\right)}$, we analyze three cases.

- If $\tilde{v}_{\mu\left(i_{1}\right)}=v_{\mu\left(i_{1}\right)}=\bar{v}_{\mu\left(i_{1}\right)}^{A}$, then, since $(\tilde{u}, \tilde{v})$ and $\left(\underline{u}^{A}, \bar{v}^{A}\right)$ belong to $C\left(w_{A}\right)$,

$$
\underline{u}_{i^{*}}^{A} \leq \tilde{u}_{i^{*}}=a_{i^{*} \mu\left(i_{1}\right)}-\tilde{v}_{\mu\left(i_{1}\right)}=a_{i^{*} \mu\left(i_{1}\right)}-\bar{v}_{\mu\left(i_{1}\right)}^{A} \leq \underline{u}_{i^{*}}^{A} .
$$


But then, by $(9), u_{i^{*}}<\tilde{u}_{i^{*}}=\underline{u}_{i^{*}}^{A}$ contradicts $(u, v) \in R^{\mu}\left(w_{A}\right)$.

- If $\tilde{v}_{\mu\left(i_{1}\right)}=v_{\mu\left(i_{1}\right)}<\bar{v}_{\mu\left(i_{1}\right)}^{A}$, define

$u_{i}^{\varepsilon}=\tilde{u}_{i}-\varepsilon$ and $v_{\mu(i)}^{\varepsilon}=\tilde{v}_{\mu(i)}+\varepsilon$ for all $i \in M$ such that $\tilde{v}_{\mu(i)}<\bar{v}_{\mu(i)}^{A}$,

$u_{i}^{\varepsilon}=\tilde{u}_{i}$ and $v_{\mu(i)}^{\varepsilon}=\tilde{v}_{\mu(i)}$ for all $i \in M$ such that $\tilde{v}_{\mu(i)}=\bar{v}_{\mu(i)}^{A}$.

Notice that, for $\varepsilon>0$ small enough $\left(u^{\varepsilon}, v^{\varepsilon}\right) \in C\left(w_{A}\right)$. Indeed, if $\tilde{v}_{\mu(i)}<\bar{v}_{\mu(i)}^{A}$, we have $\tilde{u}_{i}>\underline{u}_{i}^{A} \geq 0$. Also, if $\tilde{v}_{\mu(i)}<\bar{v}_{\mu(i)}^{A}$ and $\tilde{v}_{j}=\bar{v}_{j}^{A}$, we prove that $\tilde{u}_{i}+\tilde{v}_{j}>a_{i j}$ and thus, for $\varepsilon>0$ small enough, $u_{i}^{\varepsilon}+v_{j}^{\varepsilon} \geq a_{i j}$. The reason is that if $\tilde{v}_{j}=\bar{v}_{j}^{A}$ and $\tilde{u}_{i}+\tilde{v}_{j}=a_{i j}$, then $\underline{u}_{i}^{A} \leq \tilde{u}_{i}=a_{i j}-\tilde{v}_{j}=a_{i j}-\bar{v}_{j}^{A} \leq \underline{u}_{i}^{A}$, where the last inequality follows from $\left(\underline{u}^{A}, \bar{v}^{A}\right) \in C\left(w_{A}\right)$. Thus $\tilde{u}_{i}=\underline{u}_{i}^{A}$, but this implies $\tilde{v}_{\mu(i)}=\bar{v}_{\mu(i)}^{A}$, in contradiction with the assumption. Moreover, since $\tilde{u}_{i^{*}}>$ $u_{i^{*}} \geq \underline{u}_{i^{*}}^{A}$, we have $\tilde{v}_{\mu\left(i^{*}\right)}<\bar{v}_{\mu\left(i^{*}\right)}^{A}$ which implies the existence of $\varepsilon>0$ small enough we so that $\left(u^{\varepsilon}, v^{\varepsilon}\right) \in C\left(w_{A}\right)$ with $u_{i^{*}}^{\varepsilon}>u_{i^{*}}, v_{\mu\left(i_{1}\right)}^{\varepsilon}>\tilde{v}_{\mu\left(i_{1}\right)}=v_{\mu\left(i_{1}\right)}$ and $u_{i^{*}}^{\varepsilon}+v_{\mu\left(i_{1}\right)}^{\varepsilon}=\tilde{u}_{i^{*}}+\tilde{v}_{\mu\left(i_{1}\right)}=a_{i^{*} \mu\left(i_{1}\right)}$. Therefore $\left(u^{\varepsilon}, v^{\varepsilon}\right) \operatorname{dom}_{\left\{i^{*}, \mu\left(i_{1}\right)\right\}}^{w_{A}}(u, v)$.

- If $\tilde{v}_{\mu\left(i_{1}\right)}<v_{\mu\left(i_{1}\right)}$, then, since both $(\tilde{u}, \tilde{v})$ and $(u, v)$ are in the $\mu$-principal section, $\tilde{u}_{i_{1}}>u_{i_{1}}$. We then repeat the argument above with the mixed pair $\left\{i_{1}, \mu\left(i_{2}\right)\right\}$. Either we find that there exists $\left(u^{\prime}, v^{\prime}\right) \in C\left(w_{A}\right)$ such that $\left(u^{\prime}, v^{\prime}\right) \operatorname{dom}_{\left\{i_{l}, \mu\left(i_{l+1}\right)\right\}}^{w_{A}}(u, v)$ for some $l \in\{1,2, \ldots, r-1\}$ or we reach $\tilde{u}_{i_{r}}>u_{i_{r}}$ and, since $\tilde{v}_{j^{*}}>v_{j^{*}}$ and $\tilde{u}_{i_{r}}+\tilde{v}_{j^{*}}=a_{i_{r} j^{*}}$, we obtain $(\tilde{u}, \tilde{v}) \operatorname{dom}_{\left\{i_{r}, j^{*}\right\}}^{w_{A}}(u, v)$.

This concludes the proof of Claim 3.1. It remains to prove external stability for elements in the $\mu$-principal section but outside the limits of individual core bounds. We do this first under some additional assumptions.

Claim 3.2. Let $\left(M \cup M^{\prime}, w_{A}\right)$ be a square assignment game and $\mu \in \mathcal{M}_{A}^{*}\left(M, M^{\prime}\right)$. If $a_{i j}>0$ for all $(i, j) \in \mu$, then any $(u, v) \in B^{\mu}\left(w_{A}\right) \backslash\left(R^{\mu}\left(w_{A}\right) \cup V^{\mu}\left(w_{A}\right)\right)$ is dominated by some element of $V^{\mu}\left(w_{A}\right)$. 
The proof of this claim is consigned to Appendix B in the supplemental material since it is rather long and technical. In this proof, among other results on assignment games, we make use of Claim 3.1 above.

Notice that this completes the stability of $V^{\mu}\left(w_{A}\right)$ for square assignment markets where all optimal pairs make a positive profit. We only need to extend this to the remaining assignment games.

Claim 3.3. Let $\left(M \cup M^{\prime}, w_{A}\right)$ be an assignment game, $\mu \in \mathcal{M}_{A}^{*}\left(M, M^{\prime}\right)$ and assume that there exists $i \in M$ such that $a_{i \mu(i)}=0$ or there exist unassigned agents. Then $V^{\mu}\left(w_{A}\right)$ is a stable set.

Proof of Claim 3.3

In this case, let $I=\left\{i \in M \mid a_{i \mu(i)}=0\right.$ or $i$ is unmatched by $\left.\mu\right\}$ and $J=\left\{j \in M^{\prime} \mid a_{\mu^{-1}(j) j}=0\right.$ or $j$ is unmatched by $\left.\mu\right\}$. With some abuse of notation, we denote by $\mu$ the restriction of $\mu$ to $(M \backslash I) \times\left(M^{\prime} \backslash J\right)$. Then, if we consider the submarket $\left((M \backslash I) \cup\left(M^{\prime} \backslash J\right), w_{A^{\prime}}\right)$ where $A^{\prime}=A_{\mid(M \backslash I) \times\left(M^{\prime} \backslash J\right)}$, we are under the assumptions of Claim 3.2 and we already know that $V^{\mu}\left(w_{A^{\prime}}\right)=$ $\bigcup_{(R, S) \in \mathcal{C}_{A^{\prime}}^{\mu}} \hat{C}\left(w_{A^{\prime}-R \cup S}\right)$ is a stable set of $\left((M \backslash I) \cup\left(M^{\prime} \backslash J\right), w_{A^{\prime}}\right)$. Notice also that $B^{\mu}\left(w_{A}\right)=\left\{\begin{array}{l|l}(u, v) \in \mathbb{R}^{M} \times \mathbb{R}^{M^{\prime}} & \begin{array}{l}\left(u_{-I}, v_{-J}\right) \in B^{\mu}\left(w_{A^{\prime}}\right), \\ u_{i}=0 \text { for all } i \in I, v_{j}=0 \text { for all } j \in J\end{array}\end{array}\right\}$.

We now claim

$V^{\mu}\left(w_{A}\right)=\left\{\begin{array}{l|l}(u, v) \in \mathbb{R}^{M} \times \mathbb{R}^{M^{\prime}} & \begin{array}{l}\left(u_{-I}, v_{-J}\right) \in V^{\mu}\left(w_{A^{\prime}}\right), \\ u_{i}=0 \text { for all } i \in I, v_{j}=0 \text { for all } j \in J\end{array}\end{array}\right\}$

and it is a stable set for the initial market $\left(M \cup M^{\prime}, w_{A}\right)$. Let us denote by $\hat{V}^{\mu}\left(w_{A^{\prime}}\right)$ the right-hand side of (10). 
To prove the above equality, notice first that $\left((M \backslash I) \cup\left(M^{\prime} \backslash J\right), w_{A_{-I \cup J}}\right)$ is a $\mu$-compatible subgame of $\left(M \cup M^{\prime}, w_{A}\right)$. Moreover, because of the definition of the sets $I$ and $J$, any $\mu$-compatible subgame of $\left((M \backslash I) \cup\left(M^{\prime} \backslash J\right), w_{A_{-I \cup J}}\right)$ is also a $\mu$-compatible subgame of $\left(M \cup M^{\prime}, w_{A}\right)$. Therefore $\hat{V}^{\mu}\left(w_{A^{\prime}}\right) \subseteq V^{\mu}\left(w_{A}\right)$. Now, if there existed $(u, v) \in V^{\mu}\left(w_{A}\right) \backslash \hat{V}^{\mu}\left(w_{A^{\prime}}\right)$, then $\left(u_{-I}, v_{-J}\right) \in I\left(w_{A^{\prime}}\right) \backslash$ $V^{\mu}\left(w_{A^{\prime}}\right)$ and the external stability of $V^{\mu}\left(w_{A^{\prime}}\right)$ would imply the existence of $\left(u_{-I}^{\prime}, v_{-J}^{\prime}\right) \in V^{\mu}\left(w_{A^{\prime}}\right)$ such that $\left(u_{-I}^{\prime}, v_{-J}^{\prime}\right) d o m^{w_{A^{\prime}}}\left(u_{-I}, v_{-J}\right)$. The usual completion of $\left(u_{-I}^{\prime}, v_{-J}^{\prime}\right)$ by giving null payoffs to agents in $I \cup J$ leads to $\left(u^{\prime}, v^{\prime}\right) \in \hat{V}^{\mu}\left(w_{A^{\prime}}\right) \subseteq V^{\mu}\left(w_{A}\right)$ which dominates $(u, v)$, in contradiction to the known internal stability of $V^{\mu}\left(w_{A}\right)$. Once obtained that $V^{\mu}\left(w_{A}\right)=\hat{V}^{\mu}\left(w_{A^{\prime}}\right)$, the internal and external stability of this set follow straightforwardly from those of $V^{\mu}\left(w_{A^{\prime}}\right)$.

Hence, we have proved that any assignment game has a von NeumannMorgenstern stable set (in fact there exists one for each optimal matching). Let us illustrate this main result with an example of assignment game proposed in Shapley and Shubik [11].

Example 3.1. Let $M=\{1,2,3\}$ be the set of buyers, $M^{\prime}=\left\{1^{\prime}, 2^{\prime}, 3^{\prime}\right\}$ be the set of sellers, and the valuation matrix be

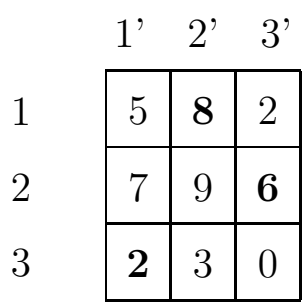

The only optimal matching is $\mu=\left\{\left(1,2^{\prime}\right),\left(2,3^{\prime}\right),\left(3,1^{\prime}\right)\right\}$ and the non-trivial $\mu$-compatible subgames $w_{A_{-I \cup J}}$ are defined by the following pairs $(I, J)$ : 


$$
\begin{array}{l|l|l}
I=\{2\}, J=\emptyset & I=\emptyset, J=\left\{1^{\prime}\right\} & I=\{2\}, J=\left\{1^{\prime}\right\} \\
I=\{2,3\}, J=\emptyset & I=\emptyset, J=\left\{1^{\prime}, 2^{\prime}\right\}
\end{array}
$$

To obtain a $\mu$-compatible subgame, usually $I$ and $J$ cannot be simultaneously non-empty. The reason is that if agents of both sides of the market are removed (and these are not unassigned agents), then their optimal partners by $\mu$ tend to become matched in the submarket and this in general contradicts the fact that the restriction of $\mu$ is an optimal matching of the submarket. There are, nevertheless, exceptions that occur either when the market is not square and one of the removed agents is an unassigned agent on the large side, or when $i \in I \cap \mu^{-1}\left(M^{\prime}\right), j \in J \cap \mu(M)$ and $a_{\mu^{-1}(j) \mu(i)}=0$, as is the case in the present example with $I=\{2\}$ and $J=\left\{1^{\prime}\right\}$, since $a_{33}=0$.

The claimed von Neumann-Morgenstern stable set (see Figure 1) is $V^{\mu}\left(w_{A}\right)=C\left(w_{A}\right) \cup \hat{C}\left(w_{A_{-\{2\}}}\right) \cup \hat{C}\left(w_{A_{-\{2,3\}}}\right) \cup \hat{C}\left(w_{A_{-\left\{1^{\prime}\right\}}}\right) \cup \hat{C}\left(w_{A_{-\left\{1^{\prime} 2^{\prime}\right\}}}\right) \cup \hat{C}\left(w_{A_{-\left\{2,1^{\prime}\right\}}}\right)$

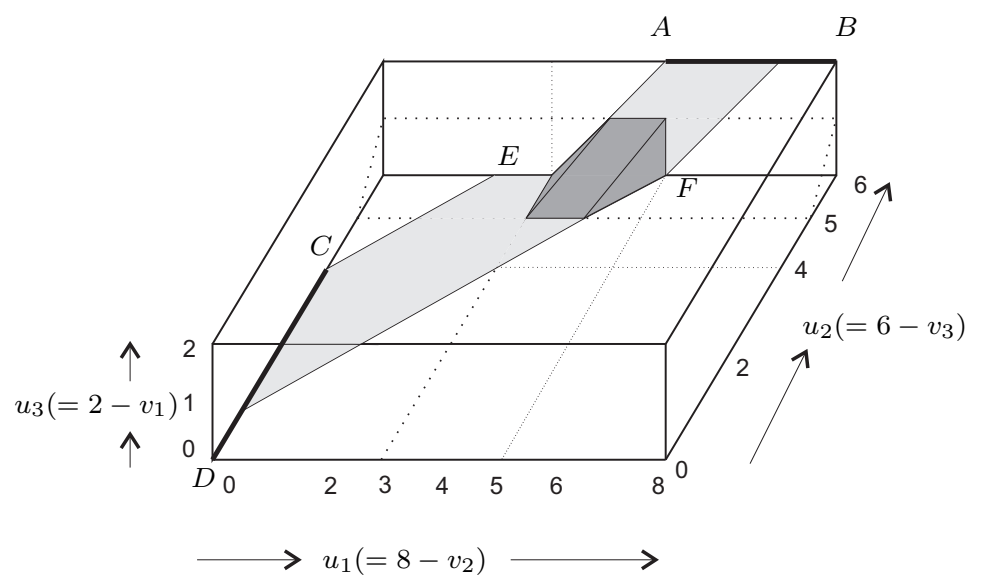

Figure 1: 
The parallelepiped $[0,8] \times[0,6] \times[0,2]$ is the projection of the principal section $B^{\mu}\left(w_{A}\right)$ to the space of the buyers' payoff. To obtain the sellers' payoffs, remember that $\mu=\left\{\left(1,2^{\prime}\right),\left(2,3^{\prime}\right),\left(3,1^{\prime}\right)\right\}$, and $u_{i}+v_{\mu(i)}=a_{i \mu(i)}$ for all $i \in M$ and $(u, v) \in B^{\mu}\left(w_{A}\right)$. Inside this parallelepiped we represent the core $C\left(w_{A}\right)$ in dark grey. The shaded area in the face $u_{2}=a_{23}=6$ is the extended core of one $\mu$-compatible subgame, $\hat{C}\left(w_{A_{-\{2\}}}\right)$; and the shaded area in the face $v_{1}=a_{31}=2$ (or $\left.u_{3}=0\right)$ is $\hat{C}\left(w_{A_{-\left\{1^{\prime}\right\}}}\right)$. The segment $[A, B]$ is $\hat{C}\left(w_{A_{-\{2,3\}}}\right)$, while the segment $[C, D]$ is $\hat{C}\left(w_{A_{-\left\{1^{\prime} 2^{\prime}\right\}}}\right)$. In this example, $\hat{C}\left(w_{A_{-\left\{2,1^{\prime}\right\}}}\right)=[E, F] \subseteq \hat{C}\left(w_{A_{-\left\{1^{\prime}\right\}}}\right)$.

\section{Concluding remarks}

The von Neumann-Morgenstern stable set $V^{\mu}\left(w_{A}\right)$ we have proved to exist for the assignment game $\left(M \cup M^{\prime}, w_{A}\right)$ is the only one in the $\mu$-principal section. The reason is that, by Shubik [12], no imputation in $V^{\mu}\left(w_{A}\right)$ can be dominated by another imputation in the $\mu$-principal section.

Proposition 4.1 (Shubik, 1984). Let $\left(M \cup M^{\prime}, w_{A}\right)$ be an assignment game and $\mu \in \mathcal{M}_{A}^{*}\left(M, M^{\prime}\right)$ an optimal matching. The only stable set that excludes third-party payments (according to $\mu$ ) is $V^{\mu}\left(w_{A}\right)$.

Such stable sets represent a quite natural standard of behavior: once an optimal matching $\mu$ has been agreed on, if some agents leave the market with the whole profit of their respective partnerships in such a way that their partners remain in the market ${ }^{6}$ and the restriction of the selected matching is

\footnotetext{
${ }^{6}$ When $a_{i \mu(i)}=0$ for some $i \in M$, both partners may leave the market.
} 
still optimal for the submarket, then the remaining agents allocate the profit of the submarket following the core principle of undomination.

There are, however, many other stable sets of the assignment game outside the $\mu$-principal sections. It is possible to find examples in markets with at most two agents on each side. Moreover, Shapley [10] describes infinitely many stable sets for the symmetric assignment games (glove markets).

All these stable sets are complete lattices ${ }^{7}$ with respect to the same partial order.

Given $(u, v),\left(u^{\prime}, v^{\prime}\right) \in I\left(w_{A}\right)$, we say $(u, v) \leq_{M}\left(u^{\prime}, v^{\prime}\right)$ if and only if $u_{i} \leq u_{i}^{\prime}$ for all $i \in M$ and $v_{j} \geq v_{j}^{\prime}$ for all $j \in M^{\prime}$. It is not difficult to prove that the stable set $V^{\mu}\left(w_{A}\right)$ is a complete lattice with respect to $\leq_{M}$, but in fact this result is more general.

Proposition 4.2. Every stable set of an assignment game is a complete lattice with respect to $\leq_{M}$.

The proof is based on the fact that no stable set of an assignment game with at least three agents contains an open set of the imputation set. To see this, we only need to follow the proof of Shapley [10] for the symmetric case.

The lattice property is something the stable sets of the assignment game have in common with the stable sets of the one-to-one matching problems (the marriage problem), as proved recently by Ehlers [2].

Finally, the present paper suggests a procedure that may be useful for obtaining stable sets for other classes of games. We may look for stable sets that are the union of the core of the game and the extended cores of certain

\footnotetext{
${ }^{7}$ See Shapley and Shubik [11] for a definition of a complete lattice in this setting.
} 
subgames. For instance, this procedure could be useful for looking for stable sets in markets with one seller who owns several objects and several buyers on the opposite side who demand one unit each, or even in the more general assignment markets (with several sellers) of Kaneko [4].

Also in the marriage problem (see Roth and Sotomayor, [9]) it would be interesting to analyze whether a stable set can be obtained by enlarging the set of stable matchings by adding stable matchings of certain submarkets. This would be even more interesting in some generalizations of the marriage market that may have an empty core, as is the case of many-to-one matching markets or three-sided matching markets, as well as in the multisided generalization of the assignment game (Quint, [8]).

\section{References}

[1] R. Aumann. What is game theory trying to accomplish? In: Frontiers of Economics, K. Arrow and S. Honkapohja eds., Oxford, 1985.

[2] L. Ehlers. Von Neumann-Morgenstern stable sets in matching problems. Journal of Economic Theory, 134 (2007) 537-547.

[3] D.B. Gillies. Solutions to general non-zero-sum games. In: Tucker, A. W.; Luce, R. D., Contributions to the Theory of Games IV. Annals of Mathematics Studies, 40(1959) 47-85.

[4] M. Kaneko. On the core and competitive equilibria of a market with indivisible goods. Naval Research Logistics Quarterly, 23 (1976) 321-337.

[5] W.F. Lucas. A game with no solution. Bulletin of the American Mathematical Society, 74(1968) 237-239. 
[6] M. Núñez, C. Rafels. Buyer-seller exactness in the assignment game. International Journal of Game Theory, 31(2002) 423-436.

[7] M. Núñez, C. Rafels. A glove market partitioned matrix related to the assignment game. Games and Economic Behavior, 67 (2009) 598-610.

[8] T. Quint. The core of an $m$-sided assignment game. Games and Economic Behavior, 3 (1991) 487-503.

[9] A. Roth, M. Sotomayor. Two-sided matching. Econometric Society Monographs, 18. Cambridge University Press, 1990.

[10] L.S. Shapley. The solutions of symmetric market games. In: Tucker, A. W. and Luce, R. D. eds., Contributions to the Theory of Games IV. Annals of Mathematics Studies, 40 (1959) 87-93.

[11] L.S. Shapley, M. Shubik. The Assignment Game I: The Core. International Journal of Game Theory, 1 (1972) 111-130.

[12] M. Shubik. A Game Theoretical Approach to Political Economy. MIT Press, Cambridge, MA, 1984.

[13] T. Solymosi, T.E.S. Raghavan. Assignment games with stable core. International Journal of Game Theory, 30 (2001) 177-185.

[14] Von Neumann, J. and Morgenstern, O. The theory of games and economic behavior. Princeton University Press, 1944. 